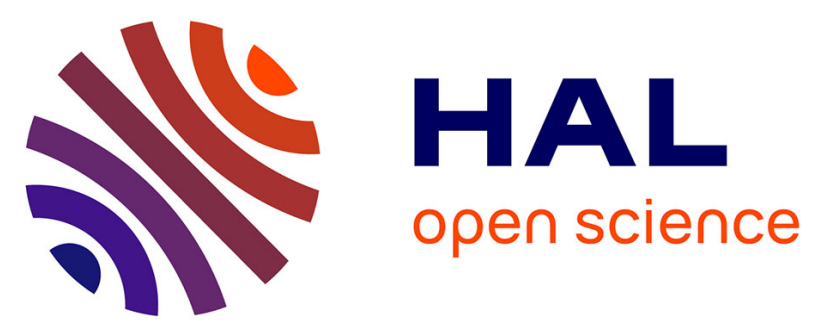

\title{
Solvent Effect on the Singlet Excited-state Dynamics of 5-Fluorouracil in Acetonitrile as Compared with Water
}

Thomas Gustavsson, Nilmoni Sarkar, Elodie Lazzarotto, Dimitra Markovitsi, Vincenzo Barone, Roberto Improta

\section{- To cite this version:}

Thomas Gustavsson, Nilmoni Sarkar, Elodie Lazzarotto, Dimitra Markovitsi, Vincenzo Barone, et al.. Solvent Effect on the Singlet Excited-state Dynamics of 5-Fluorouracil in Acetonitrile as Compared with Water. Journal of Physical Chemistry B, 2006, 110, pp.12843 -12847. 10.1021/jp062266j . hal-00083605

\section{HAL Id: hal-00083605 https://hal.science/hal-00083605}

Submitted on 5 Jul 2006

HAL is a multi-disciplinary open access archive for the deposit and dissemination of scientific research documents, whether they are published or not. The documents may come from teaching and research institutions in France or abroad, or from public or private research centers.
L'archive ouverte pluridisciplinaire $\mathbf{H A L}$, est destinée au dépôt et à la diffusion de documents scientifiques de niveau recherche, publiés ou non, émanant des établissements d'enseignement et de recherche français ou étrangers, des laboratoires publics ou privés. 


\section{Solvent effect on the singlet excited state dynamics of}

\section{5-fluorouracil in acetonitrile as compared to water}

Thomas Gustavsson*, Nilmoni Sarkar ${ }^{a}$, Elodie Lazzarotto, Dimitra Markovitsi,

Laboratoire Francis Perrin, DSM/DRECAM/SPAM - CNRS URA 2453, CEA Saclay, F-91191 Gif-surYvette, France

Vincenzo Barone $e^{\#}$ and Roberto Improta ${ }^{\#} *$

\#Dipartimento di Chimica, Universita Federico II, Complesso Universitario Monte S. Angelo, Via Cintia, I-80126 Napoli, Italy and Istituto Biostrutture e Bioimmagini /CNR, V. Mezzocannone 6 -80134 Napoli, Italy

Corresponding author email : thomas.gustavsson@cea.fr

RECEIVED DATE (automatically inserted by publisher)

Title running head : Femtosecond fluorescence and TDDFT study of 5-fluorouracil in acetonitrile

a Permanent Address : Department of Chemistry, Indian Institute of Technology, Kharagpur, PIN 721 302, WB, India. 
Abstract. The excited state dynamics of 5-fluorouracil in acetonitrile has been investigated by femtosecond fluorescence upconversion spectroscopy in combination with quantum chemistry TD-DFT calculations ((PCM/TD-PBE0). Experimentally it was found that when going from water to acetonitrile solution the fluorescence decay of 5FU becomes much faster. The calculations show that this is related to the opening of an additional decay channel in acetonitrile solution since the dark $\mathrm{n} / \pi^{*}$ excited state becomes near degenerate with the bright $\pi / \pi^{*}$ state, forming a conical intersection close to the FranckCondon region. In both solvents a $S_{1}-S_{0}$ conical intersection, governed by the out-of-plane motion of the fluorine atom is active, allowing an ultrafast internal conversion to the ground state.

\section{Key Words.}

DNA bases, pyrimidine bases, time-resolved fluorescence, fluorescence upconversion, TDDFT density functional calculations, conical intersections, 
Introduction. There is currently a keen interest in characterizing the electronically excited states of the DNA bases. These are known to undergo extremely fast non-radiative deactivation, but the underlying mechanisms remained largely unknown up to very recently. Rapid advances in time-resolved spectroscopic techniques and excited state quantum mechanical calculations have now made it possible to get fundamental insights in their excited state dynamics, both in the gas phase and in aqueous solution. ${ }^{1-5}$ However, most recent studies explain the ultrafast decay on purely intramolecular grounds, only marginally affected by solvent effects. The role played by environmental effects in the excited state dynamics is instead far from being completely assessed. The large majority of the ultrafast studies have been performed in aqueous solution, whereas the number of comparative studies in different solvents is still very limited. ${ }^{6-8}$ In these investigations, the excited state deactivation of DNA bases is still found to be ultrafast, but modulated by $\pm 50 \%$ with respect to aqueous solution, depending on the base and the solvent. Crespo-Hernandez et al. summarized solvent effects on the singlet excited states of DNA bases as modest. ${ }^{9}$ It should be noted though, that in none of these studies were the experimental results accompanied by any theoretical calculations.

In this communication we show how the nature of the solvent significantly modulates, by a factor of four, the excited state lifetime of a pyrimidine nucleobase. More precisely, 5-fluorouracil (5FU, inset in Figure 2) has been investigated in acetonitrile, a polar but aprotic solvent, by femtosecond fluorescence upconversion. The experimental observations are compared with recent results concerning different uracils in aqueous solution ${ }^{10}$ and interpreted with the aid of quantum chemistry TD-DFT calculations ((PCM/TD-PBE0). From a purely theoretical point of view, obtaining a reliable description of the relaxed excited state geometry of a large organic molecule by TD-DFT calculations including solvent effects is today only at its beginning. ${ }^{1,10-12}$

Experimental details. 5FU was purchased from Sigma Aldrich. Acetonitrile (Merck UV spectroscopic grade) was used without further purification. Absorption and fluorescence spectra were recorded with a Perkin Lamda 900 spectrophotometer and a SPEX Fluorolog-2 spectrofluorometer 
respectively. The femtosecond fluorescence upconversion setup used has been described earlier. ${ }^{13}$ It uses the frequency-tripled output at $267 \mathrm{~nm}$ from a Ti:S laser for excitation. The Gaussian apparatus function (fwhm $\approx 310 \mathrm{fs}$ ) allows a time resolution of about $100 \mathrm{fs}$ after deconvolution. Total fluorescence decays shown below were constructed from the parallel and perpendicular signals $\left(I_{\text {par }}(t)\right.$ and $\left.I_{\text {perp }}(t)\right)$ according to $I(t)=I_{p a r}(t)+2 I_{\text {perp }}(t)$. All upconversion measurements were performed at room temperature $\left(20 \pm 1{ }^{\circ} \mathrm{C}\right)$ under aerated conditions. Solutions $\left(\approx 2.5 \times 10^{-3} \mathrm{~mol} / \mathrm{dm}^{-3}, 25 \mathrm{ml}\right)$ were kept flowing through a $0.4 \mathrm{~mm}$ quartz cell, which itself was kept in continuous motion perpendicular to the excitation beam.

Computational Details. Ground state and excited state geometry optimizations on 5FU in acetonitrile were performed by DFT (PCM/PBE0) and TD-DFT (PCM/TD-PBE0) calculations. These allowed the determination of the relative energy in the Franck-Condon region and the calculation of the minima of the two lowest energy excited states deriving from the bright HOMO/LUMO $\pi / \pi^{*}$ and the dark HOMO1/LUMO $n / \pi^{*}$ transitions (hereafter $S_{\pi}$ and $S_{n}$ ). Conical Intersections $(C I)$ between the ground and the $\pi / \pi^{*}$ excited state were located at the CASSCF(8/8)/6-31G(d) level, by using the method of Bearpark et al., ${ }^{14}$ including $6 \pi$ molecular orbitals and the two $\mathrm{n}_{\mathrm{O}}$ valence orbitals. The PCM/TD-PBE0 calculations allowed ground- and excited- state geometry optimization including solvent effects. All the calculations have been performed by using a development version of the Gaussian package. ${ }^{15}$ Further details regarding the calculations are given elsewhere. ${ }^{10}$ Bulk solvent effect on the excited state has been calculated by using the PCM/TD-DFT implementation, based on the linear response (LR) theory, described in ref. 16. In some cases we have also applied the new State Specific (SS) implementation of TD-DFT methods, that should allow for a more balanced treatment of the $n / \pi^{*}$ and $\pi / \pi^{*}$ transitions. ${ }^{17}$ In brief, LR methods avoid the calculation of the exact excited state electron density in favor of a direct determination of excitation energies. On the other hand, in the SS procedure the excited state electron 
density and the corresponding response surface charges (characterizing the PCM method) are selfconsistently optimized by means of an iterative procedure.

Several papers show that PCM alone is fully adequate to model polar solvents as $\mathrm{CH}_{3} \mathrm{CN}$ without including explicit solvent molecules. ${ }^{18-21}$ The proper description of solvent shifts in aqueous solutions requires instead also the explicit inclusion of water molecules belonging to the first solvation shell. ${ }^{1}$ Taking into account experimental suggestions ${ }^{22}$, all the PCM calculations of 5FU in water solution included four explicit water molecules in the first solvation shell. ${ }^{10}$ Our model is confirmed by the results of a recent MD study of 5-fluorouracil, where no indication of hydrogen bonding between the fluorine atom and water hydrogens was found. ${ }^{23}$ Here we only treat the diketo form of 5FU. This is the most stable form in the electronic ground state, ${ }^{24,25}$ and the only tautomer identified in solution and in the gas phase. ${ }^{4,26}$

Results and discussion. Steady-state absorption and fluorescence spectra of 5FU in acetonitrile and aqueous solution are shown in Figure 1. Both absorption and fluorescence spectra are shifted toward shorter wavelengths in acetonitrile as compared to water, indicating a weaker solute-solvent interaction in the excited state. Corresponding fitted peak frequencies are given in Table 1 (see SI for details). The fluorescence quantum yield of 5FU in acetonitrile is about four times lower than in water. The most striking difference is that the fluorescence spectrum of 5FU in acetonitrile is much broader than in water, with a short wavelength flank that is displaced by $25 \mathrm{~nm}$ towards the blue while the red side is nearly superposable with that observed in water.

(Figure 1)

Fluorescence decays at $330 \mathrm{~nm}$ of 5-fluorouracil in $\mathrm{H}_{2} \mathrm{O}$ and $\mathrm{CH}_{3} \mathrm{CN}$ are shown in Figure 2. It is quite apparent that the decay in acetonitrile is much faster than that in water. We performed merged nonlinear fitting/deconvolution processes using mono- or bi-exponential impulse response model functions $i(t)$ convoluted by the Gaussian instrument response function, $I(t) \propto i(t) \otimes G(t)$. Fitted values are given in Table 2. Although mono-exponential fits are not perfect, such fits give easily comparable characteristic 
times, having the values of 1.4 and 0.4 ps in $\mathrm{H}_{2} \mathrm{O}$ and $\mathrm{CH}_{3} \mathrm{CN}$, respectively. These values are in line with the relative fluorescence intensities mentioned above.

(Figure 2)

The Vertical Excitation Energy (VEE) of the $S_{\pi}$ state (mainly arising from the HOMO-LUMO excitation, with $\pi / \pi^{*}$ character $)^{1,12}$ provided by PCM/TD-PBE0/6-311+G(2d,2p) calculations on a PCM/PBE0/6-31G(d) optimized geometry in acetonitrile solution is $39.700 \mathrm{~cm}^{-1}$, to be compared with a value of $39.400 \mathrm{~cm}^{-1}$ obtained in aqueous solution. Computed VEE's are thus in good agreement with experimental data. Even if the $\pi / \pi^{*}$ VEE is slightly overestimated (by $\sim 2000 \mathrm{~cm}^{-1}$ ), it is noteworthy that our calculations provide a very good estimate of the water $\rightarrow$ acetonitrile solvent blue-shift. The experimental value $\left(300 \mathrm{~cm}^{-1}\right)$ is indeed very close to its PCM/TD-PBE0 counterpart.

In agreement with previous computational studies on uracils in gas phase and in aqueous solution, $, 9,10$ both LR and SS PCM/TD-PBE0 calculations predict that in the FC region a dark state $\left(\mathrm{S}_{\mathrm{n}}\right)$ has a similar stability to $S_{\pi}$. The $S_{n}$ electronic transition has a predominant HOMO-1 $\rightarrow$ LUMO character $\left(\mathrm{n} / \pi^{*}\right)$, mainly involving the C4-O8 carbonyl group.

Figure 3

The structure of the $S_{\pi}$ state optimized in acetonitrile (see Figure 3) is very similar to that obtained in aqueous solution and already described in ref. 10. The pyrimidine ring adopts a "boat-like" conformation, with $\mathrm{N} 3$ and $\mathrm{C} 6$ atoms out of the average plane defined by N1, C2, C4, and C5 atoms. The largest variations of the bond lengths involve the lengthening of C4O8 and, especially, C5C6 bond distances in line with the bonding/antibonding character of HOMO and LUMO with respect to those bonds. $^{1,10}$

The computed fluorescence energy is $28.500 \mathrm{~cm}^{-1}$ to be compared to a value of $30.100 \mathrm{~cm}^{-1}$ in aqueous solution. The comparison with the experimental results is not straightforward, since the observed fluorescence spectrum of 5FU in acetonitrile is abnormally broad and the lifetime is much shorter than in water. For acetonitrile the main part of the fluorescence could thus come from the 
Franck-Condon region and the excited state population be quenched before it reaches the $S_{\pi}$ local minimum.

The stabilities of the $S_{\pi}$ and the "dark" $S_{n}$ excited states are comparable: in particular, state specific PCM/TD-PBE0/6-311+G(2d,2p) calculations provide 0-0 transition energies of 37000 and $36700 \mathrm{~cm}^{-1}$ for the $S_{\pi}$ and the $S_{n}$ state, respectively. PCM/TD-PBE0 geometry optimizations predict that the equilibrium geometry of $\mathrm{S}_{\mathrm{n}}$ is planar, as the ground state. The most relevant geometry shifts with respect to the ground state geometry involves instead the C4O8 and C5C6 bond lengths that increase by $0.1 \AA$ and $0.04 \AA$, respectively. The $\mathrm{n} / \pi^{*}$ transition involves indeed the transfer of an electron from the orbital corresponding mainly to the O8 lone pair to a $\pi^{*}$ orbital localized mainly on the C5C6 and C4O8 bonds. Except for the loss of planarity, the geometry shifts of the $S_{n}$ minimum are thus quite similar to that of the $S_{\pi}$ minimum. The latter exhibits a longer C5C6 bond distance, since the $\pi$ HOMO has a bonding C5C6 character; the former has a longer C4O8 bond distance, due to the involvement of the O8 lone pair. On the balance, however, it is likely that, at least in the first instants after excitation to the $S_{\pi}$ state, the geometry shifts induced by the absorption also lead to a stabilization of the close lying $S_{n}$ state. In fact, preliminary PCM/TD-PBE0 calculations along a one-dimensional path leading from the FC region to the $S_{\pi}$ minimum indicates the presence of crossing between $S_{\pi}$ and $S_{n}$ states.

These results suggest that the dynamical behavior of the $S_{\pi}$ state could be influenced by the $S_{n}$ state, especially in the proximity of the Franck-Condon region corresponding to a planar geometry. In this respect, it is worth noticing that resonant Raman experiments on uracil show that all but one of the vibrational modes that are more strongly affected by the electronic transition involve in-plane stretching and bending ${ }^{27,28}$, suggesting that, soon after excitation to the $S_{\pi}$ state, uracil-like molecules keep the planar geometry characteristic of the Franck-Condon region.

In order to better investigate this point, we have optimized the geometry of the $S_{\pi}$ state under the constrain of planarity: it is noteworthy that now the $S_{\pi}$ state is just $0.3 \mathrm{eV}$ more stable than $S_{n}$ (solvent fully equilibrated with the $S_{\pi}$ state) and that the difference between the energy of the two states is just 
$0.1 \mathrm{eV}$, when considering the equilibrium solvation energy of $\mathrm{S}_{\mathrm{n}}$. The presence of a conical intersection between these two states in this region is thus likely, and solvent fluctuations could act as coupling modes between $S_{\pi}$ and $S_{n}$. As a matter of fact, CASSCF calculations predicts that a Conical Intersection between $S_{n}$ and $S_{\pi}$ states (hereafter $\mathrm{CI}^{\mathrm{n} / \pi}$ ) does exist in vacuo for a geometry close to planarity. The structure of $\mathrm{CI}^{\mathrm{n} / \pi}$ is very similar to that found for uracil in the gas phase by Matsika at the CASSCF level, ${ }^{5,29}$ and PCM/TDPBE0 calculations confirm that this $\mathrm{CI}$ is present also in $\mathrm{CH}_{3} \mathrm{CN}$ solution, since the energy difference between the two states is only 0.09 eV (PCM/TD-PBEO 6-31G(d) calculations).

The main process governing the ultrafast internal conversion from $S_{\pi}$ to the $S_{0}$ ground state is the conical intersection $\mathrm{CI}^{\mathrm{S} 1 / \mathrm{S} 0}$. Confirming our previous results concerning aqueous solution, also in acetonitrile the reaction path on $\mathrm{S}_{\pi}$ is dominated by a pyramidalisation of C5 and an out-of-plane motion of the 5-substituent ( $\phi$ dihedral), leading to the $\mathrm{CI}^{\mathrm{S} 1 / \mathrm{S} 0}$. In order to ascertain if solvent affects the energy barrier possibly present on the $S_{\pi}$ potential energy surface (PES) when moving from the FC region towards $\mathrm{CI}^{\mathrm{S} 1 / \mathrm{S} 0}$, we have performed a fully relaxed energy scan of the $\mathrm{S}_{\pi}$ state as a function of the out of plane motion of the Fluoro substituent ( $\phi$ dihedral) in $\mathrm{CH}_{3} \mathrm{CN}(5 \mathrm{~F})$ and in water $\left(5 \mathrm{~F} \bullet 4 \mathrm{H}_{2} \mathrm{O}\right)$, by performing excited state PCM/TD-PBE0 geometry optimizations for fixed values of the $\phi$ dihedral. The resulting curves are shown in Figure 4.

\section{(Figure 4)}

The picture obtained in the two solvents is very similar. The curves exhibit a very shallow minimum for $\phi=170^{\circ}$, i.e. the value corresponding to the $S_{\pi}$ energy minimum and rise slowly up to $\phi=140^{\circ}$. After this point, the energy of the $S_{\pi}$ state drops and the geometry starts approaching that of the CI, where TD-PBE0 geometry optimizations suffer from severe convergence problems. Nevertheless, a partially relaxed single-point calculation for $\phi=135^{\circ}$ confirms that the energy of $S_{\pi}$ decreases with respect to $\phi=140$ suggesting that this point is a saddle point on the isomerization path.

The computed energy barriers on the $S_{\pi}$ state surface, separating the Franck-Condon region from the $\mathrm{CI}^{\mathrm{S} 1 / \mathrm{S} 0}$ conical intersection, are very similar: $0.13 \mathrm{eV}$ in $\mathrm{CH}_{3} \mathrm{CN}$ and $0.15 \mathrm{eV}$ in $\mathrm{H}_{2} \mathrm{O}$ (PCM/TD-PBE0/6- 
$31 \mathrm{G}(\mathrm{d})$ calculations). At the $6-311+\mathrm{G}(2 \mathrm{~d}, 2 \mathrm{p})$ level the energy barrier increases to $0.15 \mathrm{eV}$ in $\mathrm{CH}_{3} \mathrm{CN}$ and to $0.165 \mathrm{eV}$ in $\mathrm{H}_{2} \mathrm{O}$. The stabilization with respect to the Franck-Condon region is ca. $0.4 \mathrm{eV}$ in both solvents.

Even if the present computational analysis does not allow excluding the possibility that the solvent can modulate also the barrier heights on the path towards the $\mathrm{CI}^{\mathrm{S} 1 / \mathrm{S} 0}$ conical intersection, the comparison of the computational results obtained for $5 \mathrm{FU}$ in acetonitrile with those obtained for water solution ${ }^{10}$ strongly suggests that it affects the $S_{\pi}$ lifetime mainly by tuning the relative energy of the $S_{\pi}$ state and the close lying $S_{n}$ dark state. The stability of $\pi / \pi^{*}$ states increases both with the polarity and, especially, the hydrogen bonding ability of the solvent, implying that in water the dynamics on $S_{\pi}$ is not influenced by $S_{n}$. In acetonitrile, instead, the $S_{n}$ and the $S_{\pi}$ states are very close (their relative energy being within $0.1 \mathrm{eV}$ ) in the region of the configuration space close to the Franck-Condon region and the $\mathrm{S}_{1}$ local minimum.

According to the picture that emerges from this study (schematically illustrated in Figure 5, an additional decay channel, very likely a conical intersection between $S_{\pi}$ and $S_{n}$, opens up for $5 F U$ in acetonitrile, leading to a decrease of its lifetime, in full agreement with experiments. The broad fluorescence spectrum of 5FU in acetonitrile is another hint of the possible involvement of an additional electronic state.

(Figure 5)

The efficiency of non-radiative decay through $\mathrm{CI}^{\mathrm{n} / \pi}$ could be even more important in the gas phase or in nonpolar solvents, where the relative stability of the $\mathrm{S}_{\mathrm{n}}$ state is larger than in $\mathrm{CH}_{3} \mathrm{CN}$. This is supported by experimental results on thymine in the gas phase indicating that following photoexcitation to the $S_{\pi}$ state, the system is trapped in a dark state. ${ }^{22}$

Further experimental and theoretical (for example, dynamical computational studies) work is obviously necessary to fully assess the precise role of the solvent in these processes. We are currently extending our ultrafast fluorescence studies to other bases and other solvent environments. 
On the balance, the present study represents a very promising step towards a deeper understanding of the microscopic mechanisms underlying the photophysical behavior of nucleic acids and their constituents, showing the potentialities of the combined application of experimental and computational methods in comparative studies of different nucleobases in different solvents.

Acknowledgement. The authors thank CNRS for financial support within the framework of the European CERC3 program "Photochemistry of Nucleic Acids" and the Campus Grid at the University Federico II (Napoli) for computer resources.

Supporting Information. Steady-state absorption and fluorescence spectra on a wavenumber scale with fits, fluorescence decays on a linear scale with fits and PCM/PBE0/6-31G(d) calculated cartesian coordinates of the $S_{0}, S_{\pi}$ and $S_{1}$ minima. This material is available free of charge via the Internet at http://pubs.acs.org. 
Table 1. Characteristic parameters of the first absorption and fluorescence bands of 5-fluorouracil in acetonitrile and water. The peak frequencies $v_{\max }$ and the Stokes shift $\Delta v$ (peak absorption minus peak fluorescence). Also given are $\pi / \pi^{*}$ vertical transition energies and fluorescence transitions. All values are in $1000 \mathrm{~cm}^{-1}$.

\begin{tabular}{lcccc}
\hline & & $v_{\max }(\mathrm{abs})$ & $v_{\max }($ fluo $)$ & $\Delta v\left(\mathrm{~cm}^{-1}\right) \times 10^{3}$ \\
\hline $\mathrm{CH}_{3} \mathrm{CN}$ & Exp & 37.9 & 29.9 & 8.0 \\
& $\mathrm{Th}$ & 39.7 & 28.5 & 8.0 \\
\hline $\mathrm{H}_{2} \mathrm{O}$ & Exp & 37.6 & 29.5 & 8.1 \\
& $\mathrm{Th}$ & 39.4 & 30.1 & 8.1 \\
\hline
\end{tabular}

Table 2. Characteristic times of the fluorescence decays of 5-fluorouracil in room-temperature acetonitrile and aqueous solutions $\left(\sim 2.5 \times 10^{-3} \mathrm{M}\right)$. Results are from bi-exponential $\left(\tau_{1}, \tau_{2}\right)$ and monoexponential fits $\left(\tau_{0}\right)$. Also given is the mean time $\left(<\tau=\alpha \tau_{1}+(1-\alpha) \tau_{2}\right)$ of the bi-exponential fit.

\begin{tabular}{lccccc}
\hline & $\alpha$ & $\tau_{1}(\mathrm{ps})$ & $\tau_{2}(\mathrm{ps})$ & $<\tau(\mathrm{ps})$ & $\tau_{0}(\mathrm{ps})$ \\
\hline $\mathrm{CH}_{3} \mathrm{CN}$ & $0.81 \pm 0.11$ & $0.26 \pm 0.04$ & $0.74 \pm 0.18$ & $0.36 \pm 0.06$ & $0.39 \pm 0.07$ \\
$\mathrm{H}_{2} \mathrm{O}$ & $0.39 \pm 0.04$ & $0.69 \pm 0.06$ & $1.74 \pm 0.05$ & $1.32 \pm 0.05$ & $1.38 \pm 0.01$ \\
\hline
\end{tabular}




\section{Figure Captions}

Figure 1. Steady-state absorption and fluorescence spectra of 5-fluorouracil in room-temperature $\mathrm{H}_{2} \mathrm{O}$ and $\mathrm{CH}_{3} \mathrm{CN}$.

Figure 2. Fluorescence decays at $330 \mathrm{~nm}$ of 5-fluorouracil in $\mathrm{CH}_{3} \mathrm{CN}$ and $\mathrm{H}_{2} \mathrm{O}\left(\approx 2.5 \times 10^{-3} \mathrm{M}\right)$ after excitation at $267 \mathrm{~nm}$. Also shown is the Gaussian apparatus function ( $\mathrm{fwhm} \approx 310 \mathrm{fs}$ ).

Figure 3: Minimum of the $S_{n}$ (a) and $S_{\pi}($ b) states, according PCM/TD-PBE0-631G(d) excited state geometry optimizations in acetonitrile solution. Some selected bond distances (in $\AA$ ) are also reported. In the ground state they are, respectively: $\mathrm{C} 4 \mathrm{O} 8=1.22 \AA$, C4C5=1.45 $\mathrm{A}, \mathrm{C} 5 \mathrm{C} 61.34 \AA$.

Figure 4. Energies of the $S_{0}$ and the $S_{\pi}$ states as a function of the out of plane motion of the 5-substituent according to PCM/TD-PBE0/6-31G(d) excited state geometry optimization in acetonitrile. For comparison, the corresponding curves in water are also shown.

Figure 5. Schematic picture of the most important processes involving the electronically excited states of 5FU in acetonitrile solution. The destabilization of the $S_{n}$ state in water solution is also schematically depicted. 


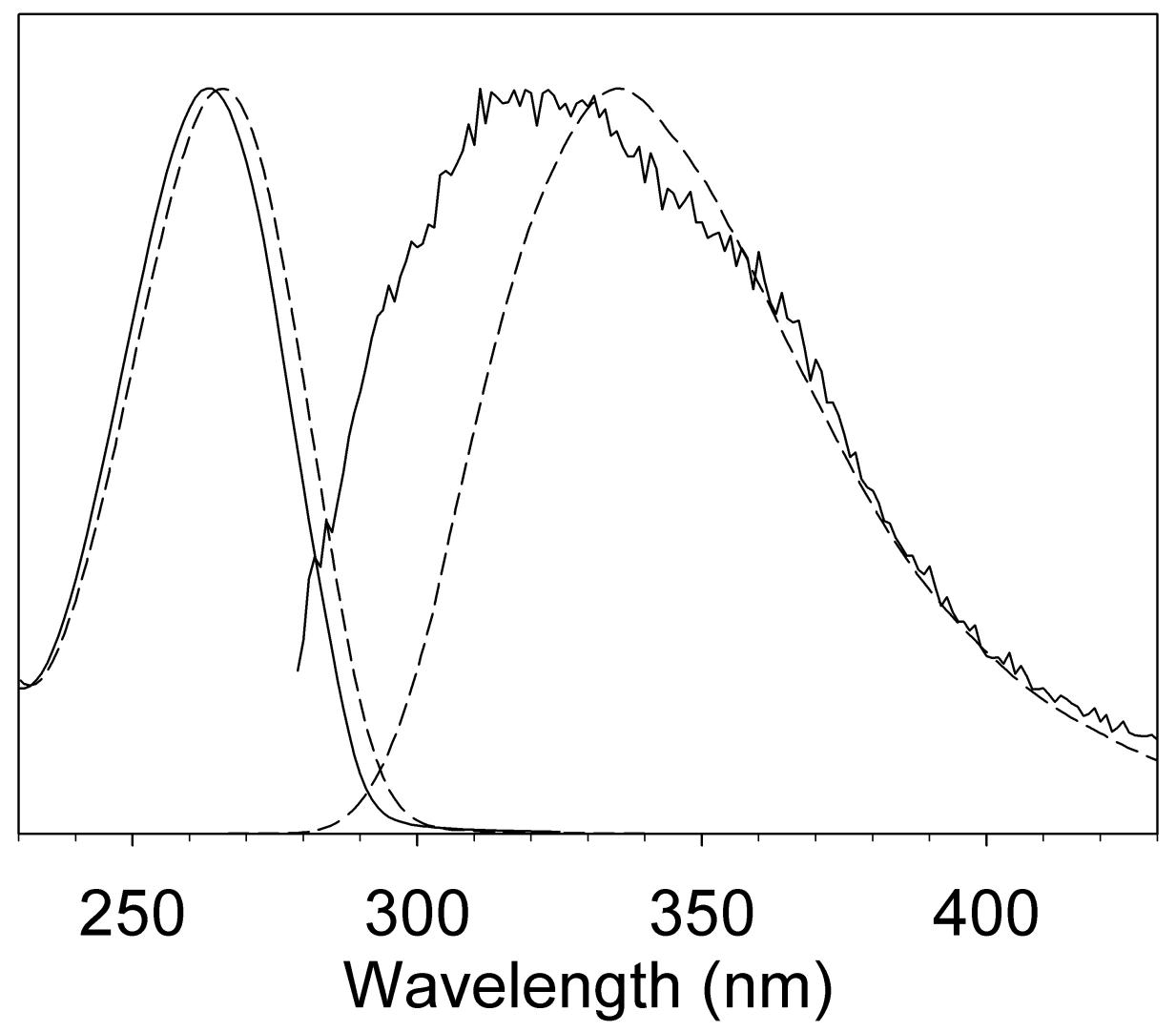

Figure 1. Steady-state absorption and fluorescence spectra of 5-fluorouracil in room-temperature $\mathrm{H}_{2} \mathrm{O}$ (dashed lines) and $\mathrm{CH}_{3} \mathrm{CN}$ (solid lines). 


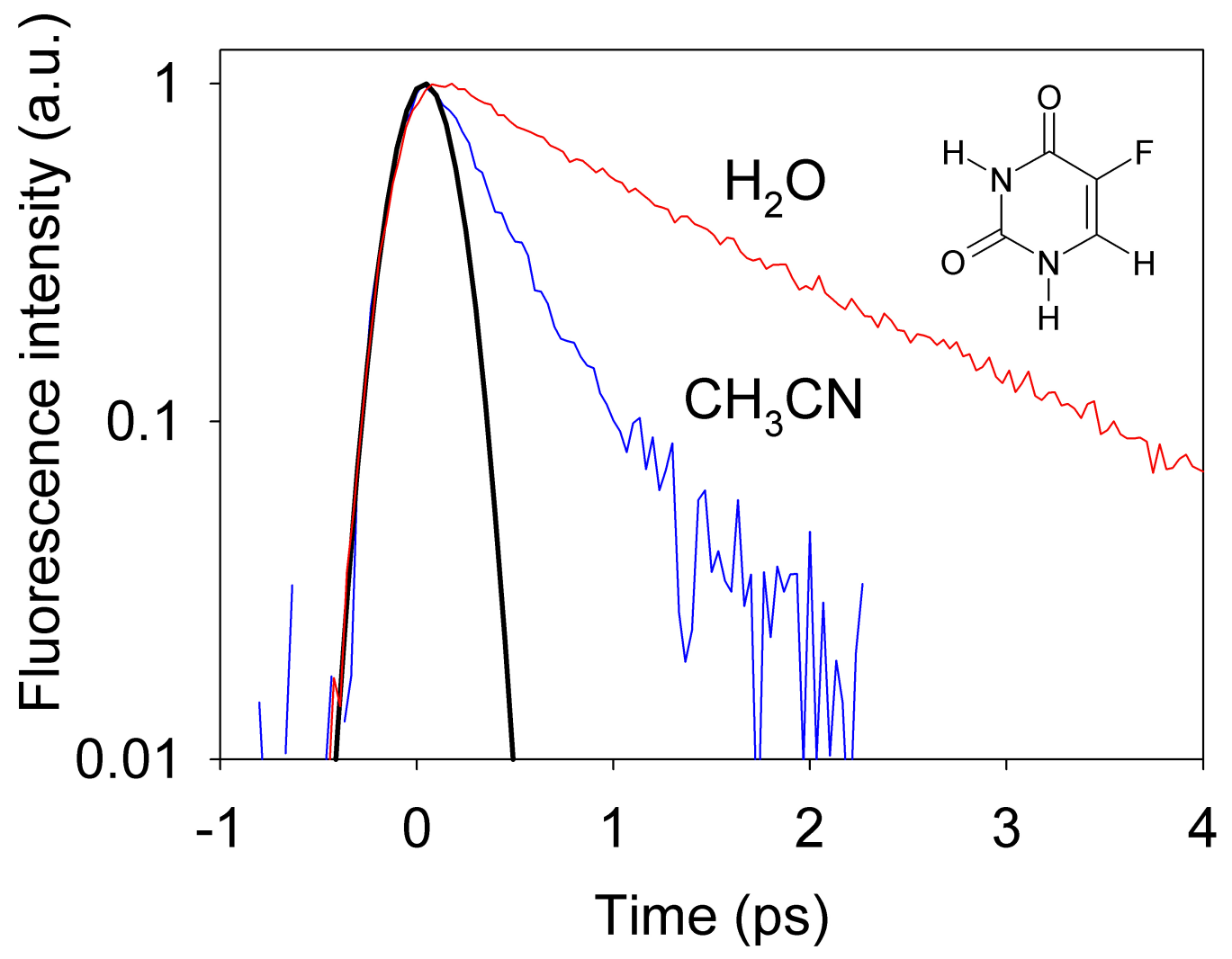

Figure 2. Fluorescence decays at $330 \mathrm{~nm}$ of 5-fluorouracil in $\mathrm{CH}_{3} \mathrm{CN}$ and $\mathrm{H}_{2} \mathrm{O}\left(\approx 2.5 \times 10^{-3} \mathrm{M}\right)$ after excitation at $267 \mathrm{~nm}$. Note that a semi-log scale is used. Also shown is the Gaussian apparatus function (fwhm $\approx 310 \mathrm{fs})$. 

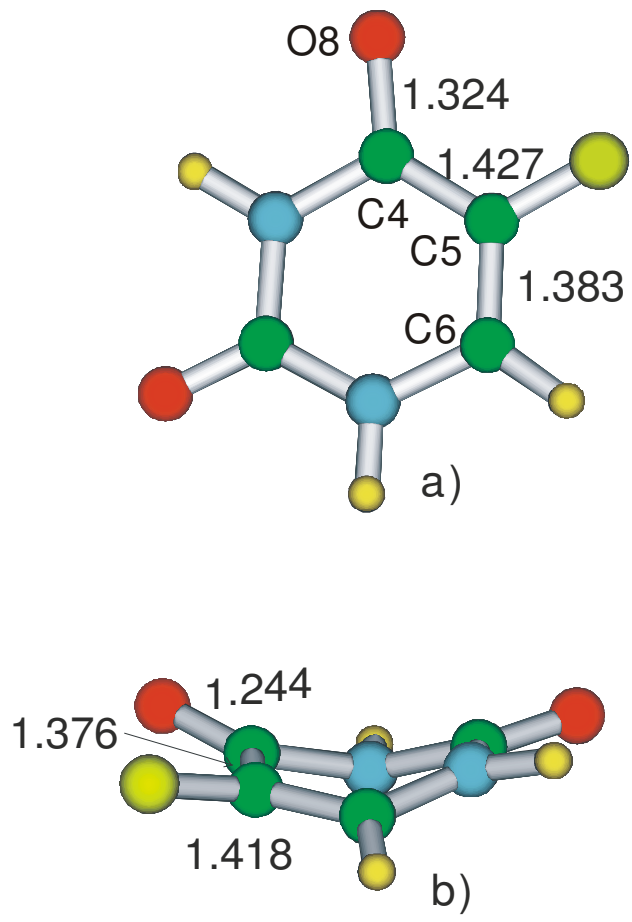

Figure 3

Figure 3: Minimum of the $S_{n}(a)$ and $S_{\pi}(b)$ states, according PCM/TD-PBE0-631G(d) excited state geometry optimizations in acetonitrile solution. Some selected bond distances (in $\AA$ ) are also reported. In the ground state they are, respectively: $\mathrm{C} 4 \mathrm{O} 8=1.22 \AA$, C4C5=1.45 $\mathrm{A}, \mathrm{C} 5 \mathrm{C} 61.34 \AA$. 


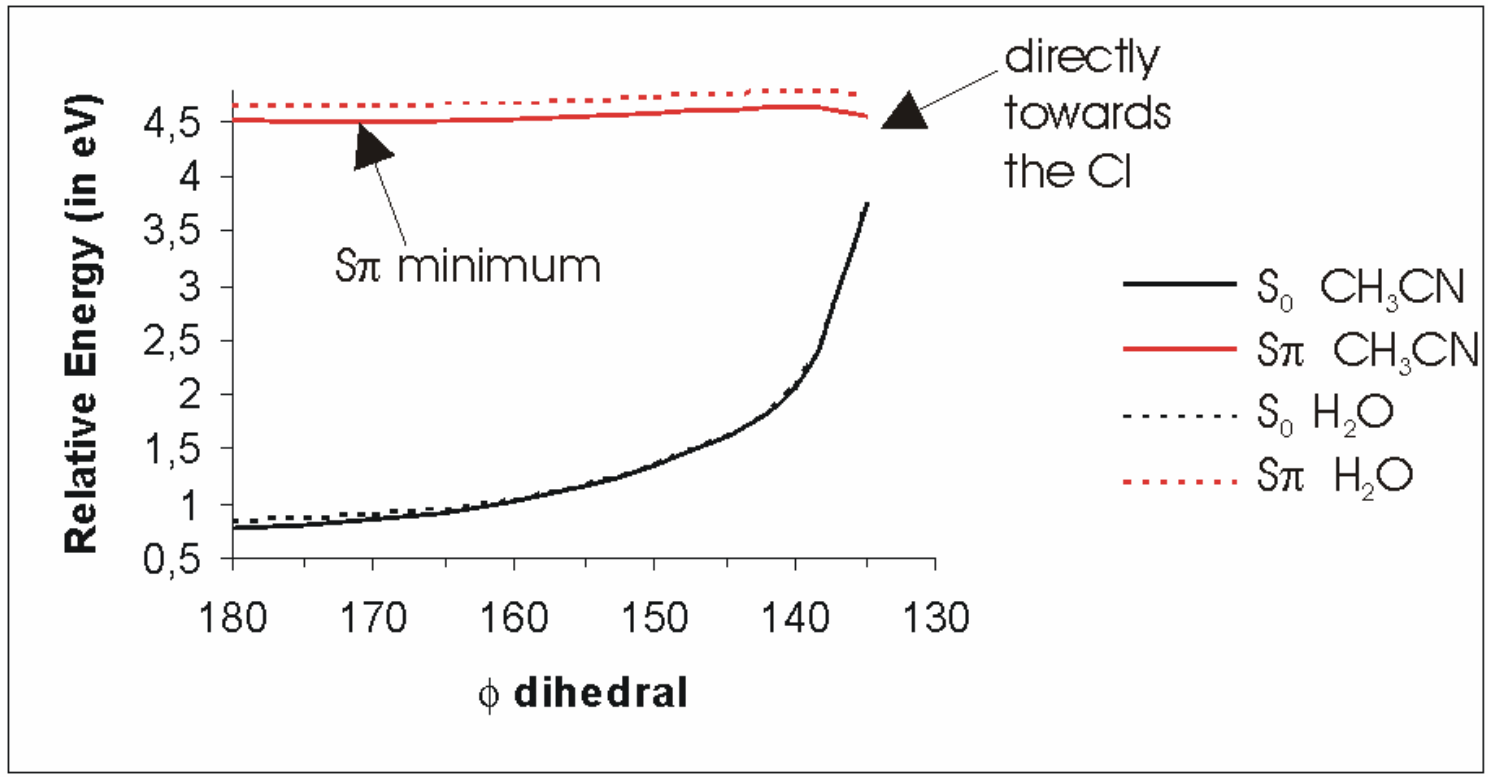

Figure 4. Energies of the $S_{0}$ and the $S_{\pi}$ states as a function of the out-of-plane motion of the 5substituent according to PCM/TD-PBE0/6-31G(d) excited state geometry optimization in acetonitrile. For comparison, the corresponding curves in water are also shown (dotted curves). 


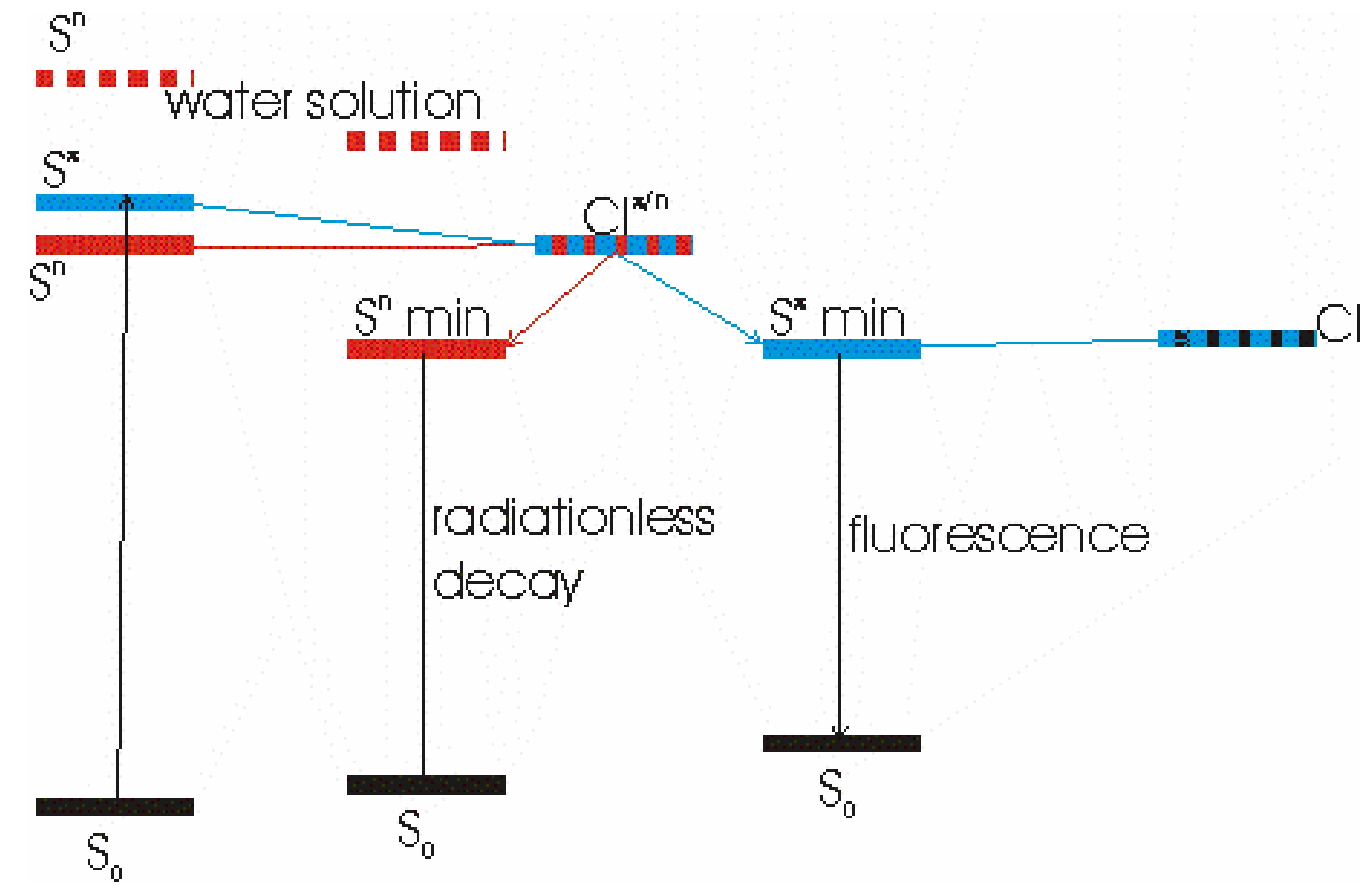

Figure 5. Schematic picture of the most important processes involving the electronically excited states of 5FU in acetonitrile solution. The destabilization of the $S_{n}$ state in water solution is also schematically depicted (dashed red lines). 


\section{References}

(1) Improta, R.; Barone, V. J. Am. Chem. Soc. 2004, 126, 14320.

(2) Canuel, C.; Mons, M.; Piuzzi, F.; Tardivel, B.; Dimicoli, I.; Elhanine, M. J. Chem. Phys. 2005, 122, 0743161.

(3) Zgierski, M. Z.; Patchkovskii, S.; Fujiwara, T.; Lim, E. C. J. Phys. Chem. A 2005, 109,

9384

(4) Markova, N.; Enchev, V.; Timtcheva, I. J. Phys. Chem. A 2005, 109, 1981.

(5) Matsika, S. J. Phys. Chem. A 2005, 109, 7538.

(6) Häupl, T.; Windolph, C.; Jochum, T.; Brede, O.; Hermann, R. Chem. Phys. Lett. 1997, 280,520 .

(7) Cohen, B.; Hare, P. M.; Kohler, B. J. Am. Chem. Soc. 2003, 125, 13594.

(8) Blancafort, L.; Cohen, B.; Hare, P. M.; Kohler, B.; Robb, M. A. J. Phys. Chem. A 2005, $109,4431$.

(9) Crespo-Hernandez, C. E.; Cohen, B.; Hare, P. M.; Kohler, B. Chem. Rev. 2004, 104, 1977

(10) Gustavsson, T.; Banyasz, A.; Lazzarotto, E.; Markovitsi, D.; Scalmani, G.; Frisch, M. J.; Barone, V.; Improta, R. J. Am. Chem. Soc. 2006, 128, 607.

(11) Jacquemin, D.; Perpete, E. A.; Scalmani, G.; Frisch, M. J.; Ciofini, I.; Adamo, C. Chem. Phys. Lett. 2006, 421, 272.

(12) Scalmani, G.; Frisch, M. J.; Mennucci, B.; Tomasi, J.; Cammi, R.; Barone, V. J. Chem. Phys. 2006, 124, 094107.

(13) Gustavsson, T.; Sharonov, A.; Onidas, D.; Markovitsi, D. Chem. Phys. Lett. 2002, 356, 49.

(14) Bearpark, M. J.; Robb, M. A.; Schlegel, H. B. Chem. Phys. Lett. 1994, 223, 269.

(15) Frisch, M. J.et al. Gaussian Development Version, Revision D.02. In Gaussian Development Version, Revision D.02; Gaussian, Inc.: Wallingford CT, 2005.

(16) Cossi, M.; Barone, V. J. Chem. Phys. 2001, 115, 4708.

(17) Improta, R.; Barone, V.; Scalmani, G.; Frisch, M. J. J. Chem. Phys. submitted.

(18) Improta, R.; Barone, V. Chem. Rev. 2004, 104, 1231.

(19) Cossi, M.; Crescenzi, O. J. Chem. Phys. 2003, 118, 8863.

(20) Begue, D.; Carbonniere, P.; Barone, V.; Pouchan, C. Chem. Phys. Lett. 2005, 416, 206.

(21) Tomasi, J.; Mennucci, B.; Cammi, R. Chem. Rev. 2005, 105, 2999.

(22) He, Y.; Wu, C.; Kong, W. J. Phys. Chem. A 2004, 108, 943

(23) Hamad, S.; Moon, C.; Catlow, C. R. A.; Hulme, A. T.; Price, S. L. J. Phys. Chem. B 2006, 110, 3323.

(24) Marian, C. M.; Schneider, F.; Kleinschmidt, M.; Tatchen, J. Eur. Phys. J. D: Atom., Mol. and Opt. Phys. 2002, 20, 357.

(25) Estrin, D. A.; Paglieri, L.; Corongiu, G. J. Phys. Chem. 1994, 98, 5653.

(26) Becker, R. S.; Kogan, G. Photochem. Photobiol. 1980, 31, 5.

(27) Peticolas, W. R.; Rush III, T. Journal of Computational Chemistry 1995, 16, 1262.

(28) Fodor, S. P. A.; Fava, R. P.; Hays, T. R.; Spiro, T. G. J. Am. Chem. Soc. 1985, 107, 1520.

(29) Matsika, S. J. Phys. Chem. A 2004, 108, 7584. 